\section{Is there any relationship between extra-pulmonary manifestations of Mycoplasma pneumoniae infection and atopy/respiratory allergy in children?}

\author{
Dimitri Poddighe,1,2 Gian Luigi Marseglia2 \\ 1Pediatric Unit, Melegnano Hospital, \\ Milan; 2Department of Pediatrics, \\ University of Pavia, Italy
}

\begin{abstract}
Mycoplasma pneumoniae is a common cause of respiratory infections in children, but sometimes extra-pulmonary diseases can be observed. The immunological mechanisms involved in these extra-respiratory complications are unknown. Here, we report a small case series of Mycoplasma-related diseases including 5 children who developed: i) aseptic meningitis; ii) urticarial rash and pericardial effusion; iii) pleural effusion with severe eosinophilia; iv) Stevens-Johnson syndrome; v) multiform erythema. Interestingly, all children were moderately to highly atopic, as a common immunologic feature.
\end{abstract}

\section{Introduction}

Mycoplasma pneumonia (MP) is a common cause of respiratory infections in children. This infectious agent can cause bronchitis and pneumonia. However, there is evidence that MP infection is often limited to the upper airways and patients can have mild respiratory manifestations, which are often misdiagnosed as viral illnesses. ${ }^{1}$ Indeed, the main clinical evidence of MP infection is sometimes only extra-pulmonary symptoms involving skin and joints in many cases. However, the involvement of other organs (e.g. central nervous system, heart, gastrointestinal system, eye, etc.) is well described. ${ }^{2}$ Here, we report a small case series including 5 cases of extra-pulmonary diseases, which were shown to be associated to an ongoing and/or recent MP infection, after an appropriate diagnostic work-up.

\section{Materials and Methods}

In the period between October 2014 and May 2015, among all children being admitted to the pediatric ward of Melegnano Hospital (Milan,
Italy), we studied 5 consecutive patients diagnosed as having MP-related extra-respiratory diseases. Such a diagnosis was made upon the exclusion of other concomitant diseases and infections in children showing only a significant titer of specific IgM against MP ( $>10 \mathrm{AU} / \mathrm{mL})$, in presence of a negative IgG specific serology $(<10 \mathrm{AU} / \mathrm{mL})$. Serum specific IgG and IgM against MP were measured by a chemiluminescent immunoassay (CLIA) performed through a Liason ${ }^{\circledR}$ XL analyzer [DiaSorin S.p.a, Saluggia (VC), Italy]. ${ }^{3}$ As shown in Table 1, all children received a first-level immunologic work-up, including blood cell count, serum immunoglobulin measurement, complement screening (C3 and C4) and lymphocyte phenotype.

\section{Case Report \#1}

Patient 1 was a 7-year-old boy who presented to our Emergency Pediatric Department because of high fever, persistent headache and vomiting. As neck rigor was observed, a lumbar puncture was performed: the cerebrospinal fluid resulted to be sub-limpid, showing a significant pleiocytosis, but both bacterial cultures and viral genome research (with particular regard to herpesviridae) were negative. However, as the parents reported some episodes of upper airway inflammation during the previous weeks, thus serology for MP was evaluated, demonstrating a high titer of specific IgM ( $>27 \mathrm{AU} / \mathrm{mL})$. Whereas the initial therapy with ceftriaxone and acyclovir did not lead to any improvement, actually the administration of intravenous clarithromycin rapidly improved patient's clinical condition.

\section{Case Report \#2}

Patient 2 was a 13-year-old girl having urticaria (which responded to anti-histamines and steroids only partially), diffuse arthralgia and dactylitis-like transient fingers edema. Therefore, the patient received several diagnostic investigations including heart ultrasound, which showed a pericardial effusion greater than $0.5 \mathrm{~cm}$. Eye examination did not show uveitis and autoantibody panel was completely negative; however, serum IgM against MP was present (20 AU/mL). After therapy with oral clarithromycin was started, a ready clinical improvement occurred.

\section{Case Report \#3}

Patient 3 was a 10 -year-old boy who was evaluated at the Pediatric Emergency Department because of acute onset of upper abdominal pain, vomiting and mild fever. Chest X-ray showed an
Correspondence: Dimitri Poddighe, Department of Pediatrics, Azienda 0spedaliera di Melegnano, Via Pandina 1, 20070 Vizzolo Predabissi (MI), Italy.

Tel.: +39.347.7241851

E-mail: dimimedpv@yahoo.it

Key words: Mycoplasma; atopy; extra-pulmonary diseases.

Contributions: DP conceived, drafted and wrote the manuscript; GLM provided important intellectual contribution to the paper.

Conflict of interest: the authors declare no potential conflict of interest.

Received for publication: 1 January 2016.

Revision received: 9 January 2016.

Accepted for publication: 11 January 2016.

This work is licensed under a Creative Commons Attribution NonCommercial 4.0 License (CC BYNC 4.0).

(C) Copyright D. Poddighe and G.L. Marseglia, 2016 Licensee PAGEPress, Italy

Pediatric Reports 2016; 8:6395

doi:10.4081/pr.2016.6395

interstitial pattern of lung inflammation associated with a very mild pleural effusion. Unexpectedly, the most relevant finding of the blood count was leukocytosis (WBC: $11750 / \mathrm{mm}^{3}$ ) with severe and absolute eosinophilia $\left(5750 / \mathrm{mm}^{3}\right)$. The clinical exam revealed no clinical signs of proliferative disease and blood test presented normal values of cellular turnover parameters (LDH: $456 \mathrm{U} / \mathrm{mL}$, b2microglobulin: $0.9 \mathrm{mg} / \mathrm{mL}$ ) and of liver and kidney function. A wide diagnostic work-up for infections and autoimmunity was performed: particularly, no autoantibody was showed and stools examination did not showed intestinal viral, bacterial or parasite infections. The only significant serology was related to MP, through a very high specific IgM titer ( $>27 \mathrm{UA} / \mathrm{mL})$. The treatment with clarithromycin led to a complete resolution of both symptoms and laboratory abnormalities: indeed, eosinophilia gradually decreased to normal values $\left(500 / \mathrm{mm}^{3}\right)$.

\section{Case Report \#4}

Patients 4 was a 10 -year-old boy who was admitted to the pediatric ward in order to receive intravenous nutritional and hydration support, because of the onset of severe ulcerative mucosal lesions involving lips and oral cavity, in addition to the anal area. Such a clinical picture was diagnosed as being Stevens-Johnson syndrome with prevalent mucosal involvement. After an 
extensive infectious work-up, there was no other significant finding than a high titer of anti-MP IgM (15 UA/mL). The clinical condition gradually improved during the therapy with clarithromycin.

\section{Case Report \#5}

Patient 5 was a 6 -year-old boy complaining of the acute and sudden onset of a mildly itchy, but diffuse skin rash. That was diagnosed as being multiform erythema. Whereas viral serology, including Epstein-Barr virus and cytomegalovirus, were negative, a significant increase of anti-MP IgM $(21 \mathrm{AU} / \mathrm{mL})$ was detected. Such an acute MP infection was treated through a 2-week course of clarithromycin, leading to the resolution of the clinical picture.

All patients were Caucasian and reported a recent history of airways inflammation during the previous 2-4 weeks: however, the personal and family history resulted negative for genetic diseases and immunodeficiency. An immunologic work-up was performed for all these patients and, interestingly, we observed a significant increase of serum IgE levels for age in all of them, indicating a condition of atopy. Moreover, three of them showed an overt respiratory allergy (patient 1: house dust mite and grass pollen; patient 2: house dust mite and Alternaria spp.; patient 5: grass pollen).

\section{Discussion and Conclusions}

Several studies have linked respiratory MP infections to asthma exacerbations and there are some evidences that MP might elicit a Th2 immune response in the bronchial system. ${ }^{4-5}$ As regards MP-related extra-pulmonary complications, although those are supposed to be immune-mediated/autoimmune phenomena, the immunological mechanism -or mechanisms - is unknown. ${ }^{6}$ Here, through the description of our case series, a potential association between this category of reactive diseases caused by MP and atopy might be highlighted.

A tendency to IgE production during the acute phase of MP respiratory infections has been previously described and specific $\operatorname{IgE}$ against this microorganism could be detected.7,8 Stelmach I et al. observed several antibody and cellular immune parameters in children affected with respiratory infections, but their data did not show a significant difference in total serum IgE levels between MP positive and negative patients. Actually, a trend toward a mild and gradual increase of IgE levels was described during the first year after mycoplasma infection. ${ }^{9}$ However, in our case series, we could observe a much greater IgE elevation in these children affected with MP-related extra-pulmonary diseases compared to the data displayed in the aforementioned study.

All these observations might raise the speculation that the predisposition to produce IgE in

Table 1. Immunologic parameters in patients with extra-pulmonary disease related to Mycoplasma pneumoniae.

\begin{tabular}{lccccc} 
Parameter & Case \#1 & Case \#2 & Case \#3 & Case \#4 & Case \#5 \\
Blood count, $\mu \mathrm{L}$ & & & & & \\
Leucocytes & 6100 & 11500 & 11750 & 9950 & 8200 \\
Neutrophils & 1700 & 5300 & 1700 & 3300 & 6100 \\
Lymphocytes & 3300 & 5300 & 3600 & 4900 & 1500 \\
Monocytes & 500 & 600 & 350 & 800 & 500 \\
Eosinophils & 600 & 300 & 5700 & 800 & $<100$ \\
Basophils & $<100$ & $<100$ & 250 & 150 & $<100$ \\
Immunoglobulins & & & & & \\
IgA, mg/dL & 143 & 162 & 155 & 181 & 93 \\
IgG, mg/dL & 810 & 864 & 1011 & 1030 & 688 \\
IgM, mg/dL & 87 & 104 & 82 & 108 & 56 \\
IgE, UA/dL & 848 & 168 & 900 & 431 & 1028 \\
\hline Complement, mg/dL & & & & & \\
C3 & 110 & 147 & 172 & 153 & 126 \\
C4 & 31 & 27 & 39 & 23.8 & 24.5 \\
Lymphocyte count, \% & & & & & \\
CD3 & 68 & 55 & 66 & 62 & n.a \\
CD4 & 37 & 36 & 36 & 31 & n.a. \\
CD8 & 17 & 23 & 23 & 21 & n.a. \\
CD19 & 15 & 25 & 21 & 17 & n.a. \\
CD16/56 & 10 & 16 & 10 & 14 & n.a. \\
\hline
\end{tabular}
stence of respiratory allergy might predispose to (or might be involved in the pathogenesis of) extra-respiratory diseases related to MP acute infections. Of course, although the method used to measure MP serology showed a good reliability ${ }^{3}$ serology diagnosis of MP infection should be cautiously considered and comparative studies will be required to support this preliminary observation.

\section{References}

1. Principi N, Esposito S. Emerging role of Mycoplasma pneumoniae and Chlamydia pneumoniae in paediatric respiratory tract infections. Lancet Infect Dis 2001;1:334-44.

2. Timitilli A, Di Rocco M, Nattero G, et al. Unusual manifestations of infections due to Mycoplasma pneumoniae in children. Infez Med 2004;2:113-7.

3. Capuano F, Grassi B, Pallavicini L, et al. New Liaison ${ }^{\circledR}$ automated immunoassays for the detection of mycoplasma pneumoniae IgG and IgM antibodies in human serum/plasma specimens. At 21st ECCMID / 27th ICC 2011, Milano (Italy), 7-10 May 2011.

4. Smith-Norowitz TA, Silverberg JI, Kusonruska M, et al. Asthmatic children have increate specific anti-Mycoplasma pneumoniae IgM but not IgG or IgE-values independent of history of respiratory tract infection. Pediatr Infect Dis J 2013;32:599603.

5. Ye Q, XU XJ, Shao WX, et al. Mycoplasma pneumoniae infection in children is a risk factor for developing allergic diseases. World Sci J 2014:986527.

6. Smith LG. Mycoplasma pneumonia and its complications. Infect Dis Clin N Am 2010;24: 57-60.

7. Nagayama Y, Sakurai N, Kojima S, Funabashi $S$. Total and specific IgE resp[onses in the acute and recovery phases of respiratory infections in children. $\mathbf{J}$ Asthma 1987;24:159-66.

8. Koh YY, Park Y, Lee HJ, Kim CK. Bronchoalveolar lavage fluid from patients with micoplasma pneumonia: implication of tendency toward increate immunoglobulin E production. Pediatrics 2001;107:39-46.

9. Stelmach I, Podsiadlowicz-Borzecka M, Grzelewski T et al. Humoral and cellular immunity in children with Mycoplasma pneumoniae infection: a 1 year prospective study. Clin Diag Lab Immunol 2005;12:124650. 